\title{
Quantitative Mapping of Phase Coexistence in Mott-Peierls Insulator during Electronic and Thermally Driven Phase Transition
}

\author{
Himanshu Madan, ${ }^{*}$ Matthew Jerry, Alexej Pogrebnyakov, Theresa Mayer, and Suman Datta* \\ Electrical Engineering Department, The Pennsylvania State University, University Park, Pennsylvania 16802, United States
}

\begin{abstract}
Quantitative impedance mapping of the spatially inhomogeneous insulator-to-metal transition (IMT) in vanadium dioxide $\left(\mathrm{VO}_{2}\right)$ is performed with a lateral resolution of $50 \mathrm{~nm}$ through near-field scanning microwave microscopy (SMM) at $16 \mathrm{GHz}$. SMM is used to measure spatially resolved electronic properties of the phase coexistence in an unstrained $\mathrm{VO}_{2}$ film during the electrically as well as thermally induced IMT. A quantitative impedance map of both the electrically driven filamentary conduction and the thermally induced bulk transition is established. This was modeled as a 2-D heterogeneous resistive network where the distribution function of the IMT

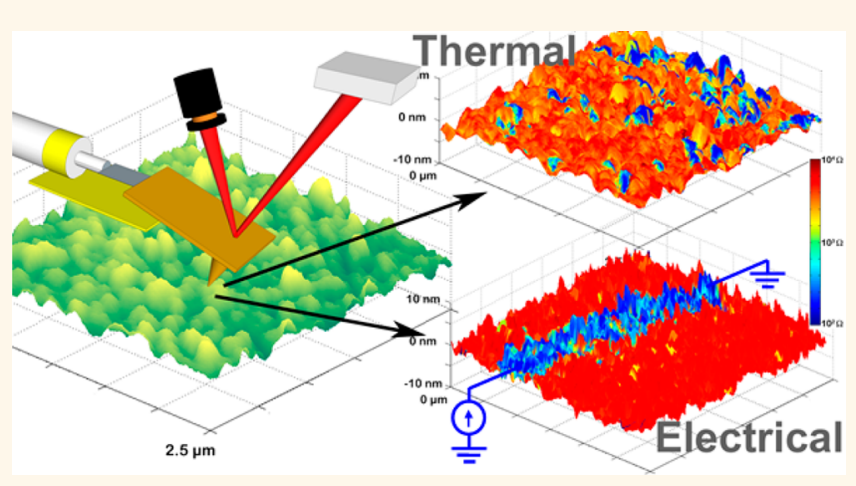
temperature across the sample is captured. Applying the resistive network model for the electrically induced IMT case, we reproduce the filamentary nature of electronically induced IMT, which elucidates a cascading avalanche effect triggered by the local electric field across nanoscale insulating and metallic domains.
\end{abstract}

KEYWORDS: vanadium dioxide - phase transition · phase coexistence - scanning microwave microscopy · insulator-to-metal transition · impedance mapping

M any solid state phase transitions involve metal-to-insulator transitions (MIT) which include a change in impedance and sometimes crystal structure. ${ }^{1-5}$ Understanding such solid state phase transitions quantitatively presents a significant research challenge owing to various factors such as high local strain and oxygen vacancies. $^{6-10}$ These inhomogeneities usually manifest as localized characteristics often at the nanoscale. ${ }^{11,12}$ Understanding the nanoscale evolution of such impedance across the phase transition is crucial not only from the paradigm of fundamental science but also to realizing practical device applications. In this work, we study a prototypical material, the transition metal oxide vanadium dioxide $\left(\mathrm{VO}_{2}\right)$. $\mathrm{VO}_{2}$ undergoes a thermally driven structural change from the monoclinic (M1) to tetragonal (rutile, R) phase with increasing temperature centered around a transition temperature $\left(T_{\mathrm{c}}\right)$ of approximately $341 \mathrm{~K} .{ }^{11}$ This transition is accompanied by a rapid change in optical properties as well as a change in the electronic conductivity over several orders of magnitude. Additionally, the insulator-to-metal transition (IMT) can be induced by mechanical, ${ }^{8}$ optical, ${ }^{13}$ or electrical stimuli. ${ }^{1}$ Further, the rapid nature of this transition, which can occur as quickly as $\sim 100 \mathrm{fs},{ }^{13}$ has sparked significant interest in the material for possible applications including, but not limited to, coupled oscillators, ${ }^{14}$ RF switches, ${ }^{15,16}$ and thermochromic devices. 8,17

As potential $\mathrm{VO}_{2}$ based device technologies scale into the decananometer regime, novel characterization techniques are required to locally probe the structural, optical, and electronic properties at the nanometer scale. This is, in part, due to the coexistence of both the metallic and insulating phases within the span of the IMT. ${ }^{12}$ For $\mathrm{VO}_{2}$, there remains much interest in imaging the domain size and unraveling the nature of the IMT in order to shed additional light on the transport mechanisms

\section{* Address correspondence to himanshu@psu.edu, sdatta@engr.psu.edu.}

Received for review December 10, 2014 and accepted January 29, 2015.

Published online January 29, 2015 $10.1021 / \mathrm{nn} 507048 d$

() 2015 American Chemical Society 
and fundamental length scales involved. Thus, spatially mapping the exact nature of the IMT at the nanoscale has been the subject of intense research through a variety of scanning techniques: scanning tunneling spectroscopy (STS), ${ }^{18}$ scanning transmission X-ray microscopy (STXM), ${ }^{19,20}$ optical microscopy, ${ }^{20,21}$ broadband near-field infrared microscopy, ${ }^{12}$ and, most recently, scanning microwave microscopy (SMM). ${ }^{22,23}$ $\mathrm{SMM}$ is a high frequency microwave scanning probe technique which measures a transformed nanoscale complex impedance at the AFM tip during a 1-port reflectivity measurement. Changes in the reflection coefficient $(\Gamma)$ are recorded at the VNA port $\left(\Gamma_{\text {port }}\right)$. The changes in $\Gamma_{\text {port }}$ are due to a change in the resonant frequency caused by variations in the local impedance beneath the SMM tip. These changes in the output signal of the SMM are recorded in two channels, $\operatorname{abs}\left(\Gamma_{\text {port }}\right)$ and phase $\left(\Gamma_{\text {port }}\right)$. Sensitivity of up to attofarad capacitances using SMM have already been demonstrated. ${ }^{24}$ However, due to the inclusion of a $\lambda / 2$ coaxial resonator, $\Gamma_{\text {port }}$ at the VNA is representative of a transformed impedance at the tip and not of the true nanoscale impedance $\left(Z_{\text {tip }}\right)$. Due to this, translation of the measured electrical data into an impedance map has proved to be a limitation, with demonstrations being limited to MOS capacitors ${ }^{25-27}$ and other silicon and silicon dioxide test structures. ${ }^{28}$ Here, we show that through a calibration of the SMM acquired microwave response using finite element modeling (FEM), an extraction of the nanoscale $R$ and $C$ values seen at the SMM tip can be realized. The nanoscale $R$ and $C$ values can then be plotted as a spatially resolved impedance map. Thus, a direct probing of the nanoscale conducting and insulating domain resistances is now possible and demonstrated during both thermal and electrically driven phase transition. In our previous work, ${ }^{19}$ STXM was used to study the evolution of the crystal structure in a $\mathrm{VO}_{2}$ film during applied bias. However, SMM offers an increased spatial resolution as well as a sensitivity derived from the true electrical properties of the film instead of exclusively the crystal structure. This results in a purely electrical characterization of the spatially mapped phase transition. Further, comparing the results of SMM impedance mapping to a 2-D heterogeneous resistive network model of the $\mathrm{VO}_{2}$ film allows for additional quantification of the mechanisms behind the phase transition, wherein the coherency of the quantitative impedance mapping and 2-D heterogeneous modeling elude to a cascading avalanche effect of transitioning nanodomains during the electrically induced phase transition and a nonuniform distribution of transition temperature across the domains in the temperature driven IMT.

\section{RESULTS AND DISCUSSION}

Structure. The structure used for this study is composed of a $100 \mathrm{~nm}$-thick $\mathrm{VO}_{2}$ film that is electrically
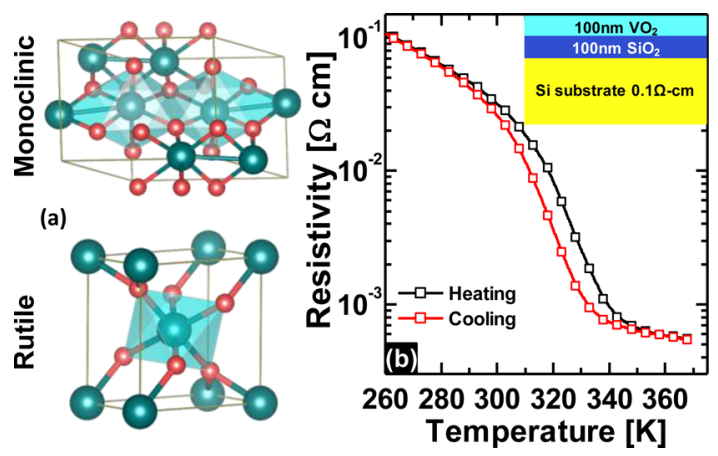

Figure 1. (a) Visualized monoclinic and rutile structures where cyan represents vanadium atoms and red oxygen atoms. (b) Resistivity vs temperature curve measured by standard van der Pauw measurement. Inset is the sample structure where $100 \mathrm{~nm} \mathrm{VO}_{2}$ is grown on $100 \mathrm{~nm} \mathrm{SiO}_{2} / 675 \mu \mathrm{m}$ Si-substrate. A doped substrate is used to provide a strong back coupling to the ground plane.

isolated from a conductive $n+$ Si substrate by a $100 \mathrm{~nm}$-thick thermally grown $\mathrm{SiO}_{2}$ layer, as shown in the inset of Figure 1b. A single-phase $\mathrm{VO}_{2}$ film was formed using a two-step process (see synthesis in Methods section). Formation of a single phase $\mathrm{VO}_{2}$ film was confirmed by X-ray diffraction (XRD) measurement, which can be seen in Figure S1 of the Supporting Information. The conducting $(0.1 \Omega \mathrm{cm})$ Si substrate is used to produce a large coupling capacitance to the ground plane, which is required for meaningful SMM imaging. The measured resistivity $v s$ temperature characteristics of the $\mathrm{VO}_{2}$ film shown in Figure $1 \mathrm{~b}$ confirms the transition temperature is near the expected value of $341 \mathrm{~K}$ for unstrained $\mathrm{VO}_{2}$. In addition, the $\mathrm{VO}_{2}$ film exhibits reversible electric-field-induced IMT switching properties as shown in Supporting Information Figure S2.

Figure $2 \mathrm{a}$ is a schematic representation of the SMM setup where an AFM with SMM nose cone is used in conjunction with a vector network analyzer (VNA). A $\lambda / 2$ resonator is required to be present in the nose cone due to the mismatch in the impedance between the characteristic $50 \Omega$ impedance of the transmission line and the large impedance seen by the SMM tip. Without the $\lambda / 2$ impedance, transformer total reflection would be measured regardless of the changes in the impedance at the tip during the imaging. With the inclusion of the $\lambda / 2$ transformer and $50 \Omega$ shunt, variations around a very large impedance at the SMM tip are converted to variations around $50 \Omega$. By establishing a resonant frequency condition of approximately every $2.57 \mathrm{GHz}$, the $\lambda / 2$ resonator allows for the highest measurement sensitivity to be located in a narrow frequency range around the $\lambda / 2$ condition. SMM utilizes this resonant frequency during a 1-port reflectivity measurement, where changes in the reflection coefficient $(\Gamma)$ are recorded at the VNA port $\left(\Gamma_{\text {port }}\right)$. The resonant frequency at $16.024 \mathrm{GHz}$ was used for all scans due to a very sharp peak allowing for a high 

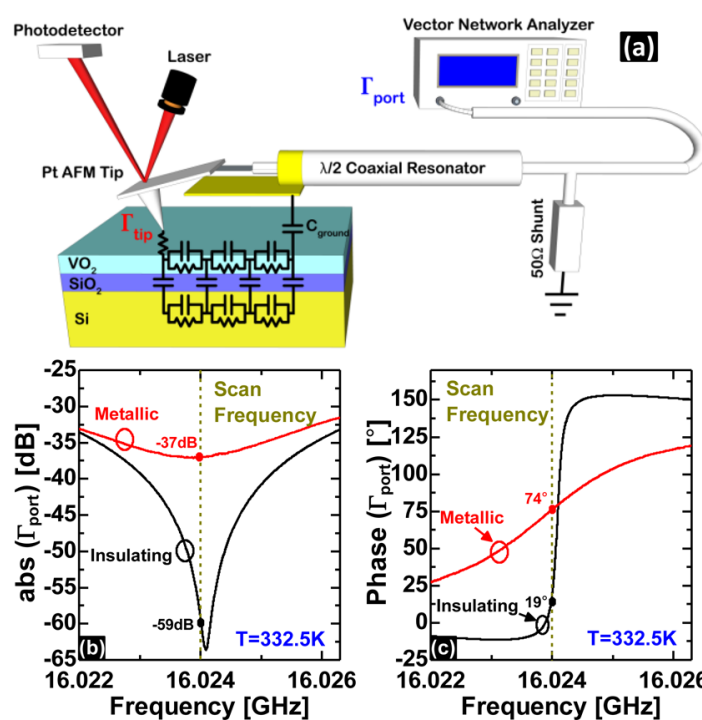

Figure 2. (a) SMM setup visualized in contact with $\mathrm{VO}_{2}$ sample. VNA is connected to a half wavelength coaxial resonator terminating at a Pt conducting AFM tip in conjunction with a $50 \mathrm{ohm}$ shunt. (b) abs $\left(\Gamma_{\text {port }}\right)$ response to insulating and conducting regions at $332.5 \mathrm{~K}$ and the corresponding (c) phase $\left(\Gamma_{\text {port }}\right)$ response. Large distinct changes between insulating and metallic domain response are observed in both traces.

dynamic range. Figure $2 b, c$ shows the SMM response to metallic and insulating domains where the large change in impedance of the domains causes dramatically different shifts in the resonant frequency. Since each scan is taken at a single frequency, large changes in the measured $\operatorname{abs}\left(\Gamma_{\text {port }}\right)$ and phase $\left(\Gamma_{\text {port }}\right)$ are recorded. Due to the inclusion of a $\lambda / 2$ coaxial resonator, $\Gamma_{\text {port }}$ at the VNA is representative of a transformed impedance at the tip and not of the true nanoscale impedance $\left(Z_{\text {tip }}\right)$, where $Z_{\text {tip }}$ is the total impedance measured at the tip to the ground plane.

Thermally Induced IMT. Scanning Microwave Microscopy (SMM) allows nanoscale imaging of the emergence and coalescence of nanoscale metallic domains as $\mathrm{VO}_{2}$ undergoes a temperature induced phase transition. The metallic phase first appears at $315 \mathrm{~K}$ and expands throughout the sample as the applied temperature progresses through the IMT. SMM imaging was performed at $2.5 \mathrm{~K}$ intervals from 315 until $352.5 \mathrm{~K}$. After every temperature step a $5 \mathrm{~min}$ wait period was taken to allow the surface temperature to reach thermal equilibrium. All scans were taken of the same $2.5 \times$ $2.5 \mu \mathrm{m}$ scan area, as seen in Figure $3 a-c$, with a resolution of $256 \times 256$. In Figure $3 d-f$ the spatial abs $\left(\Gamma_{\text {port }}\right)$ response is plotted documenting a subset of the IMT. The red regions are those of the conducting domains, while the blue regions are the insulating domains. As the temperature is increased, the coverage fraction of the metallic phase begins to approach total coverage and reaches the maximum possible coverage at a temperature slightly beyond $353 \mathrm{~K}$. The $\mathrm{SMM}$ imaging of the phase coexistence in $\mathrm{VO}_{2}$ yields
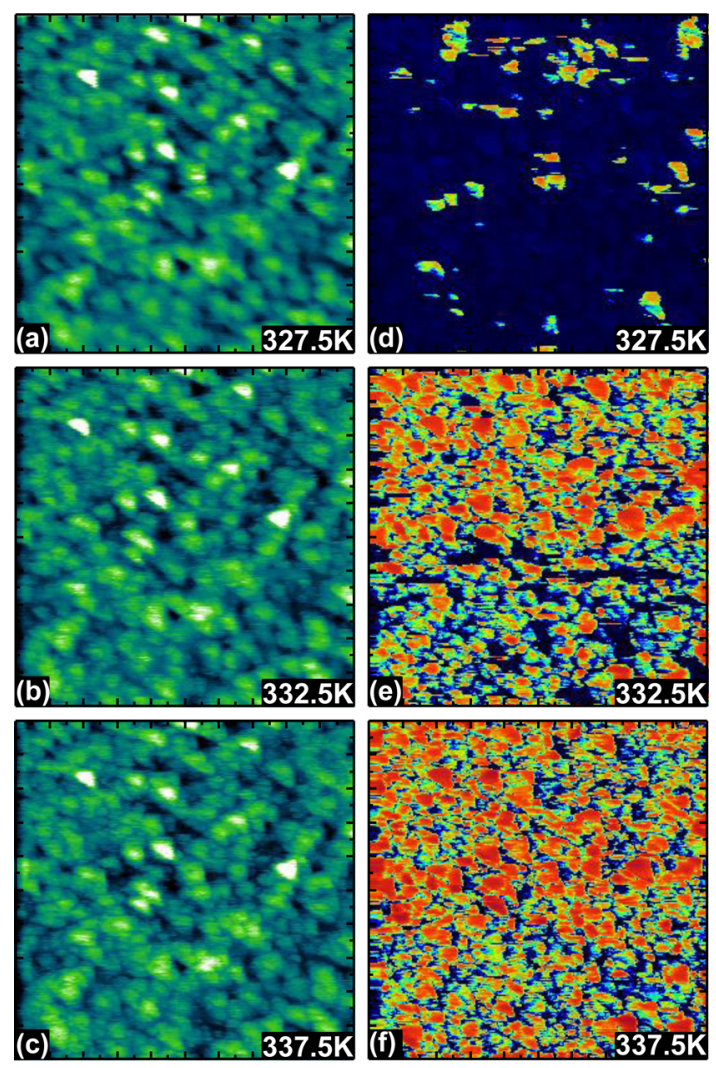

Onm

$6 \mathrm{~nm} 0 \mathrm{~dB}$

$30 \mathrm{~dB}$

Figure 3. $(\mathrm{a}-\mathrm{c})$ Normalized AFM topography of a $2.5 \mu \mathrm{m} \times$ $2.5 \mu \mathrm{m}$ size scan region at $327.5,332.5$, and $337.5 \mathrm{~K}$, respectively. Lighter regions are peaks, while dark regions are valleys within the surface topography; maximum range of surface topography is $6 \mathrm{~nm}$. (d-f) Corresponding abs$\left(\Gamma_{\text {port }}\right)$ for each of the given temperatures. Expansion of the metallic phase (red) is observed, while the (blue) insulating phases diminish with increasing temperature.

results that are consistent with previous measurements and established models for the IMT. ${ }^{12,18}$ A full set of the SMM scans can be found in the Supporting Information and all scans were found to be repeatable. Here it is observed that throughout the IMT transition nanoscale metallic domains appear within the insulating matrix and with increasing temperature reach a threshold radius of approximately $100 \mathrm{~nm}$ before coalescing to form larger regions. The observed phase coexistence is indicative of the film being comprised of nanoscale domains with varying transition temperatures owning to defects, composition, and local strain. This observation motivates modeling the film as a 2-D heterogeneous domain network which is representative of the macro-scale response seen in SMM.

Figure $4 \mathrm{a}$ shows the 2-D heterogeneous resistive network model where each nanoscale $\mathrm{VO}_{2}$ domain is taken as a node with four perpendicularly placed resistors. Each cell is representative of a single nanoscale domain, which when placed together in an $m \times n$ network describes the behavior of the macroscale sample. In accordance with the measured domain size of $\sim 100 \mathrm{~nm}$, a single domain is taken to be $100 \mathrm{~nm}$ in 

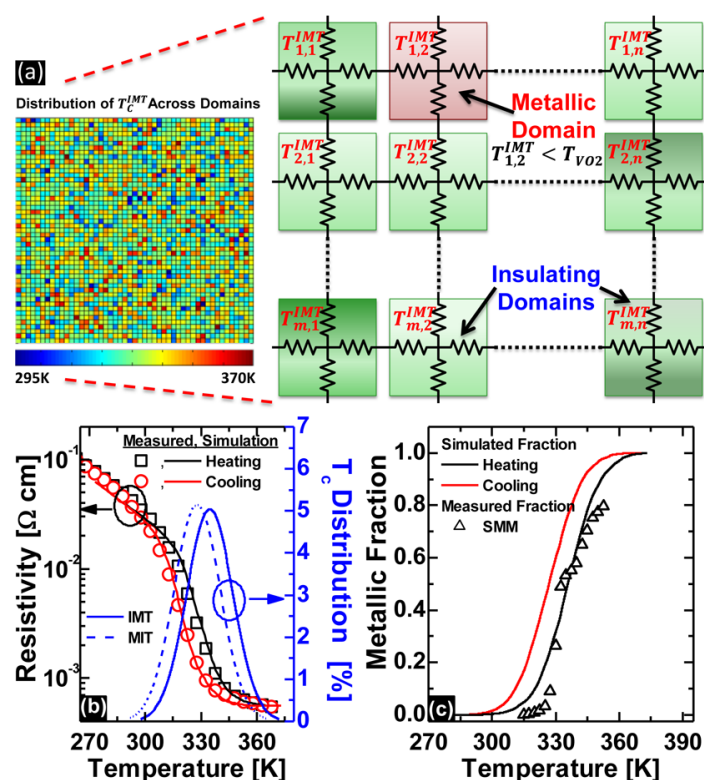

Figure 4. (a) Nanoscale domain network used to model $\mathrm{VO}_{2}$. The network consists of $m \times n$ domains each with a specific insulator to metal transition temperature, $T_{i, j}^{\mathrm{MT}}$. (b) Comparison of simulated and measured resistivity. (c) Comparison of simulated and SMM measured metallic domain fraction. The SMM metallic domain fraction was calculated by weighted average of the measured abs $\left(\Gamma_{\text {port }}\right)$ response.

length and width. As such, the model was run at $50 \times$ 50 domains, modeling an area of $5 \mu \mathrm{m} \times 5 \mu \mathrm{m}$. Each node can be in the insulating or metallic state. The insulating state resistivity is defined by eq $1^{29}$ where $E_{\mathrm{a}}$ is the activation energy $(0.19 \mathrm{eV})$, and $\rho_{1}$ is a fitting parameter $\left(1.29 \times 10^{-5} \Omega \mathrm{cm}\right)$.

$$
\rho_{\mathrm{m} 1}(T)=\rho_{1} \exp \left(\frac{E_{\mathrm{a}}}{k_{\mathrm{B}} T}\right)
$$

And the metallic state resistivity $\rho_{\mathrm{r}}$ is $5.53 \times 10^{-4} \Omega \mathrm{cm}$. By assuming that each cell in the network has a specific transition temperature, $T_{i, j}^{\mathrm{MT}}$ and $T_{i, j}^{\mathrm{MIT}}$, and distributing the transition temperatures of the individual domains across a Gaussian distribution centered on the macroscopic $T_{c}^{\mathrm{MT}}$ and $T_{c}^{\mathrm{MIT} 30}$ (distributions are shown on the Y2-axis in Figure $4 \mathrm{~b}$ as wells as Figure $4 \mathrm{a}$ ), we can reproduce the insulator to metal transition. In the model, a domain is considered to undergo a metal to insulator transition once the temperature of the sample has exceeded the transition temperature $\left(T_{i, j}^{\mathrm{MT}}\right)$ of the given domain $i, j$ (for heating). After each temperature, the resistivity is calculated from the resistance which comes from solving the potential at each node in the domain network as described in Poklonski et al. ${ }^{31}$ The simulated resistivity is then plotted against the physically measured values shown in Figure 4b. Figure $4 c$ shows the metallic fraction (percentage of total domains that are in the metallic state) computed during the simulation as a function of temperature. This is overlaid upon the metallic fraction taken directly from SMM measurements. The SMM measured fraction is taken from $\operatorname{abs}\left(\Gamma_{\text {port }}\right)$ and was calculated by a weighted scaling of the measured $\operatorname{abs}\left(\Gamma_{\text {port }}\right)$, where the maximum value was considered to be completely metallic and the lowest value was considered to be completely insulating. The intermediate values were taken to be linearly proportional to metallic fraction. The strong correlation between the measured and simulated metallic fractions as well as measured and simulated resistivity indicates that the assumption of a 2-D heterogeneous resistive network is reasonable.

To further use SMM to characterize the nanoscale domain structure of the thermally driven IMT in $\mathrm{VO}_{2}$, the $\operatorname{abs}\left(\Gamma_{\text {port }}\right)$ and phase $\left(\Gamma_{\text {port }}\right)$ response must be transformed to true nanoscale impedance as seen by the tip in reference to the ground plane $\left(Z_{\text {tip }}\right)$. This has proved to be a major hurdle that has been the subject of recent research in calibration of the $a b s\left(\Gamma_{\text {port }}\right)$ and phase $\left(\Gamma_{\text {port }}\right)$ that are collected at the VNA port. A successful calibration of abs $\left(\Gamma_{\text {port }}\right)$ and phase $\left(\Gamma_{\text {port }}\right)$ will yield a spatial map of the resistance and capacitance seen locally at the SMM tip. To relate $\Gamma_{\text {port }}$ to $Z_{\text {tip }}$, we employ a standard single-port VNA calibration where three known impedances from a model are used to solve a nonlinear system of equations. This begins by relating $\Gamma_{\text {port }}$ and $\Gamma_{\text {tip }}$ in the following equations. ${ }^{27,32}$

$$
\begin{gathered}
\Gamma_{\text {port }}=e_{00}+\frac{\Gamma_{\text {tip }} e_{01}}{1-e_{11} \Gamma_{\text {tip }}} \\
\Gamma_{\text {tip }}=\frac{Z_{\text {tip }}-Z_{\text {ref }}}{Z_{\text {tip }}+Z_{\text {ref }}}
\end{gathered}
$$

Equation 2 is a complex nonlinear equation with three unknowns, namely, $e_{00}, e_{01}, e_{11}$. To solve eq 2 , three known values of $\Gamma_{\text {tip }}$ must be taken from a model. COMSOL multiphysics modeling ${ }^{33}$ was used to determine three impedances for calibration on each scan in Figure 3. These three known impedances can be related to $\Gamma_{\text {tip }}$ by eq 3 . To accurately model the $\mathrm{VO}_{2}$ and the underlying $\mathrm{SiO}_{2} /$ Silicon substrate, impedance spectroscopy was performed vertically between patterned gold pads and a grounded chuck at multiple temperatures. A COMSOL model was designed at the full scale of the sample and the material values were tuned to fit the measured impedance spectroscopy results and determine correct parameters for the underlying substrate. These fitting results can be found in the Supporting Information. The model showed that the leakage current through the oxide and substrate increased slightly with temperature, however the values largely remained the same and did not affect the output impedance more than a tenth of a percent between the scans. With the use of the calibrated COMSOL model, three values of the $\mathrm{VO}_{2}$ impedance were simulated where resistivity was taken corresponding to an average resistivity at the scan temperature, metallic state resistivity, and insulating state, resistivity. The complex nonlinear system of equations 
in 2 were then solved to evaluate $e_{00}, e_{01}$, and $e_{11}$ at each scan temperature in Figure $1 \mathrm{~b}$. Once $e_{00}, e_{01}$, and $e_{11}$ are obtained, they are then used to calibrate the impedance map by solving eq 4 at each point of the map from the $\operatorname{abs}\left(\Gamma_{\text {port }}\right)$ and phase $\left(\Gamma_{\text {port }}\right)$ to evaluate $\Gamma_{\text {tip. }}$. From eq $5, \Gamma_{\text {tip }}$ can be converted to a local impedance at the tip. ${ }^{27,32}$

$$
\begin{gathered}
\Gamma_{\text {tip }}=\frac{\Gamma_{\text {port }}-e_{00}}{e_{01}+e_{11}\left(\Gamma_{\text {port }}-e_{00}\right)} \\
Z_{\text {tip }}=Z_{\text {ref }} \frac{1+\Gamma_{\text {tip }}}{1-\Gamma_{\text {tip }}}
\end{gathered}
$$

$Z_{\text {ref }}$ is an arbitrarily chosen reference impedance for satisfaction of eqs 3 and 5 ; a value of $10 \mathrm{k} \Omega$ is chosen as was done in ref27, but any value will suffice. This calibration results in an impedance seen by the tip in reference to the ground plane and therefore includes the local material properties at an effective radius of $43 \mathrm{~nm}$ under the tip as well material properties from the underlying substrate on the signal path to ground. $C_{\text {tip }}$ will be strongly influenced by the tip geometry, substrate capacitance, and fringing electric field. However, the value of $R_{\text {tip }}$ resulting from the calibration is relatively immune to these effects and will largely represent the $\mathrm{VO}_{2}$ film resistivity. The resulting calibrated impedance maps are shown in Figure $5 \mathrm{a}-\mathrm{f}$. Further, COMSOL modeling reveals that $R_{\text {tip }} \approx R_{\mathrm{VO} 2}$ within an error range of $1-23 \%$ with the greatest accuracy occurring in the metallic regions. The $C_{\text {tip }}$ value however is primarily due to the $\mathrm{SiO}_{2}$ and the underlying $\mathrm{Si}$ substrate. In Figure $5 \mathrm{a}-\mathrm{c}$, the blue regions correspond to the metallic domains and the red pertain to the insulating regions. In the $C_{\text {tip }}$ map, the red regions are the conducting domains and exhibit a larger capacitance to the large area with which the signal is capacitively coupled to the silicon substrate.

Electrically Induced IMT. The use of SMM to study the IMT in $\mathrm{VO}_{2}$ has implications extending into the device characterization. Here SMM can be utilized for in situ mapping of an electrically induced phase transition. To image the IMT induced by an electrical stimulus, two terminal devices were patterned on the $\mathrm{VO}_{2}$ film as can be seen in Figure 6a. The devices were lithographically defined with a channel length of $6 \mu \mathrm{m}$ and width of $20 \mu \mathrm{m} . \mathrm{Pd}(20 \mathrm{~nm}) / \mathrm{Au}(60 \mathrm{~nm})$ contacts were deposited through e-beam evaporation. To bias the devices during scanning, the contact pads were extended far away from the device to accommodate the size of the SMM nose cone. The contact pads were then wire bonded to a standard chip carrier package which was subsequently connected to a Keithley 4200 semiconductor characterization system. The temperature of the device was raised from room temperature to $315 \mathrm{~K}$ to allow switching to occur at lower voltages as seen in Supporting Information Figure S2. A current vs applied
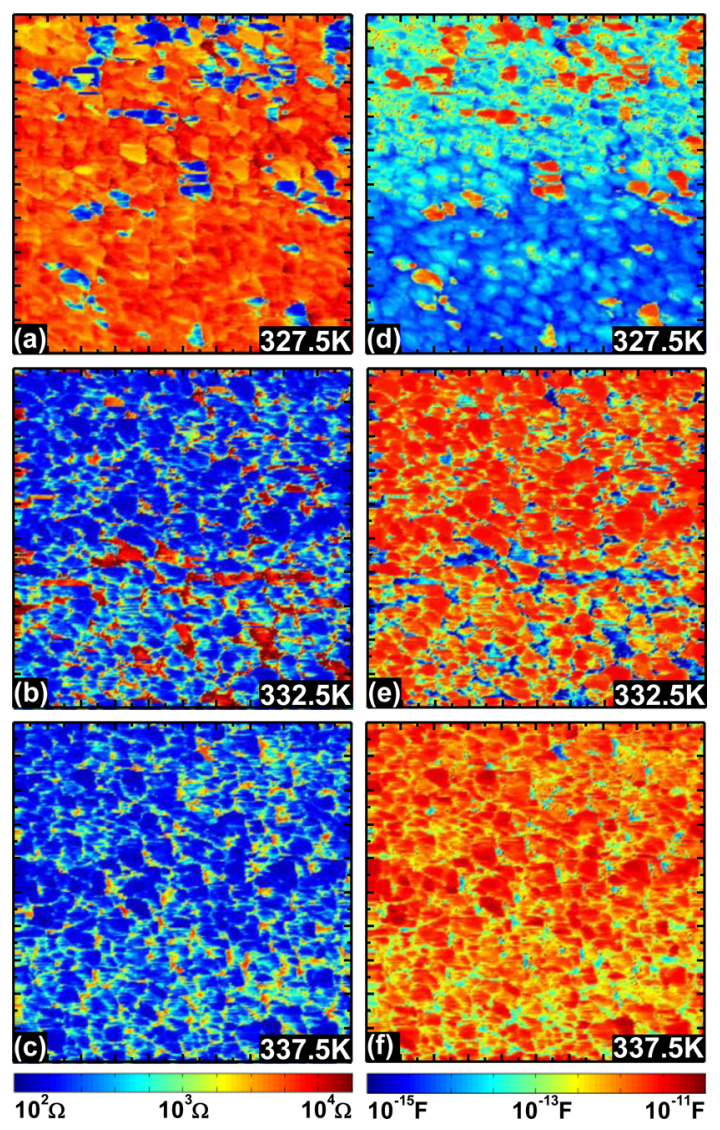

Figure 5. $(\mathrm{a}-\mathrm{c})$ Extracted $R_{\text {tip }}$ values from eqs 6 and 7. Blue regions represent the metallic phase and hence exhibit resistances of around $130 \Omega$, while the red insulating regions are approximately $7000 \Omega$. (d--) Are the extracted $C_{\text {tip }}$ values which are smaller for the insulating domains as compared to the metallic domains. This results from the capacitance being primarily due to the $\mathrm{SiO}_{2}$ layer and the conducting regions provide a larger area from which to couple to the Si substrate.

electric field sweep is shown in Figure $6 \mathrm{~b}$ where it can be seen that the device switches at an electric field of $12 \mathrm{kV} / \mathrm{cm}$; a current compliance was set to $20 \mathrm{~mA}$. SMM scans were then performed at individual current input biases from $3.25 \mathrm{~mA}$ to $20 \mathrm{~mA}$ in order to spatially resolve the IMT within the device channel. This was performed by biasing the device with a Keithly 4200 semiconductor parameter analyzer set in current mode to the desired current value $(3.25-20 \mathrm{~mA})$ for the duration of the scan. As the current bias was applied between the contact pads, nonuniform conduction across the channel is identified within $a b s\left(\Gamma_{\text {port }}\right)$ and phase $\left(\Gamma_{\text {port }}\right)$ of the SMM response. It can be clearly seen from Figure $6 c-f$ that a metallic filament appears first within the insulating matrix as the conduction path. It is further observed that as the current is increased the filament expands and eventually consumes the entire width of the channel of the device. This dependence of filament width to the applied input bias can be seen in Figure $7 \mathrm{~b}$. Below the threshold switching current, 3.25 $\mathrm{mA}$ in this case, no filament formation is seen within the device channel. 


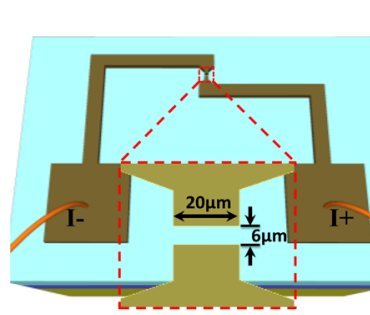

(a)
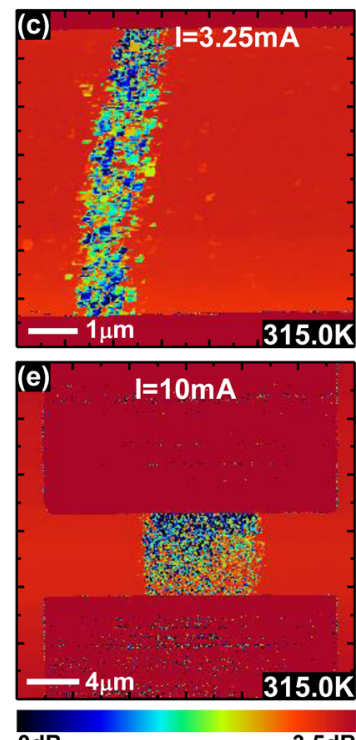

OdB

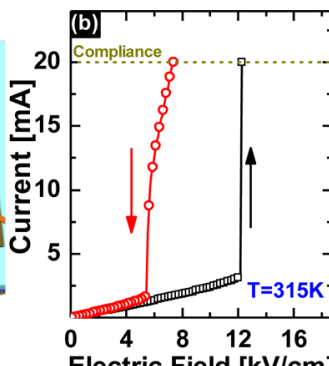

Electric Field $[\mathrm{kV} / \mathrm{cm}]$
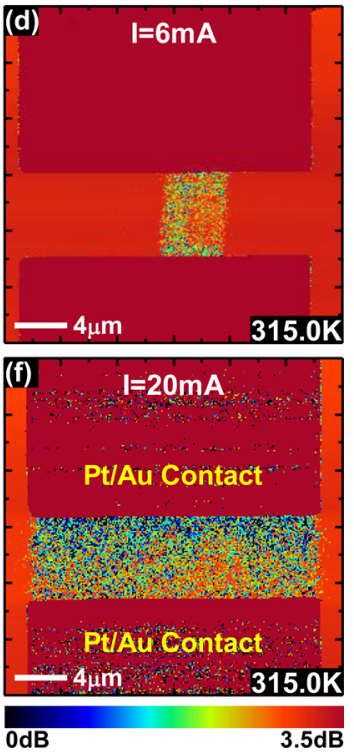

Figure 6. (a) Device schematic for SMM imaging of the electrically induced IMT. (b) Current vs electric field sweep take of the device under test at $315 \mathrm{~K}$. (c-f) In situ abs(Г) maps of electrically induced phase transition. A thin conductive filament forms across the channel and expands with the applied current. (c) The smallest achievable conducting filament which forms at $3.25 \mathrm{~mA}(7 \mu \mathrm{m} \times 7 \mu \mathrm{m})$. (d-f) abs( $\Gamma)$ maps show the growth of the filament as the device is subjected to higher currents $(24 \mu \mathrm{m} \times 24 \mu \mathrm{m})$.

$\operatorname{Abs}\left(\Gamma_{\text {port }}\right)$ and phase $\left(\Gamma_{\text {port }}\right)$ maps are transformed into an impedance map by the process described earlier. An in situ nanoscale mapping of the resistance characterizing the conducting filament is shown in Figure 7a. To fit the observed filament width dependence on the applied current, a uniformity factor, $\beta$, needs to be introduced. The uniformity factor provides the percentage of metallic domains that are present in the volume of the measured filament in order to account for the inhomogeneous nature of the measured filament. The filament width $\left(W_{\text {fil }}\right)$ is given by eq 6.

$$
\mathrm{W}_{\mathrm{fil}}=\frac{\frac{J W}{E}-\frac{W}{\rho_{\mathrm{i}}}}{\frac{\beta}{\rho_{\mathrm{m}}}-\frac{1}{\rho_{\mathrm{i}}}+\frac{1-\beta}{\rho_{i}}}
$$

Where $J$ is the current density, $\rho_{\mathrm{m}}$ and $\rho_{\mathrm{i}}$ are the metallic and insulating domain resistivity, respectively, $L$ and $W$ are the physical device dimensions, and $E$ is the applied field. $\beta$ is a unitless factor from 0 to 1 which is extracted
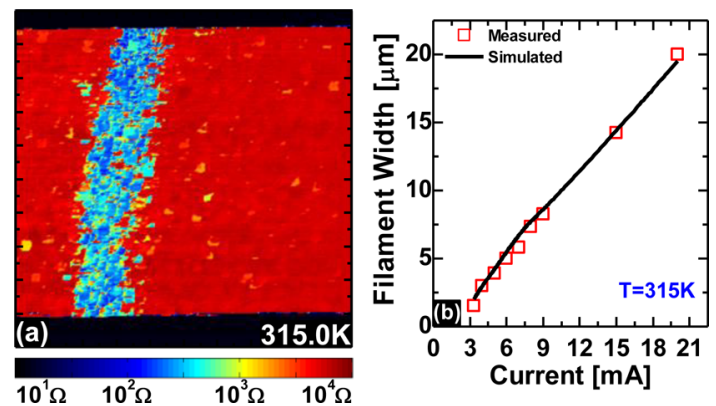

Figure 7. (a) Extracted resistance map of Figure $6 \mathrm{c}(7 \mu \mathrm{m} \times$ $7 \mu \mathrm{m})$. The blue color corresponds to conducting $\mathrm{VO}_{2}$ regions which form a small $1.3 \mu \mathrm{m}$ wide filament spanning the device channel. The red regions corresponds to the remaining insulating portion of the channel. The black regions at the top and bottom are that of the highly conductive Au contacts. (b) The measured dependence of filament widths as a function of applied input current bias.

from the imaged filament by means of a weighted average. Extracted values for $\beta$ are within the range of $0.27-0.39$ for all scans. A $\beta$ of 0.35 is used in eq 6 to fit the measured filament width in Figure $7 \mathrm{~b}$. This demonstrates the ability of SMM to directly image the nanoscale conduction within scaled $\mathrm{VO}_{2}$ based switches.

Furthermore, the 2-D heterogeneous resistive network model can be used to simulate the $\mathrm{VO}_{2}$ sample under an applied field. This is schematically illustrated in Figure 8a where one end of the sample is grounded while the other is set to the applied voltage. Again the assumption is made that each domain can individually switch between the insulating to metallic states. As such, the probability of a domain undergoing an IMT is given by eq $7 .{ }^{34}$

$$
P_{\mathrm{IMT}}=\mathrm{e}^{-\left(E_{\mathrm{B}}-q \Delta V / \gamma\right) / k T}
$$

Here, $\Delta V$ is the average voltage drop across the domain, $E_{\mathrm{B}}$ is the energy barrier between the stable insulating and metallic states, and $\gamma(0.35)$ is a geometric factor to account for the simulation grid size. Figure $8 \mathrm{~b}$ shows how $\Delta V$ is calculated, while Figure $8 \mathrm{c}$ shows how $\Delta V$ is used to modulate the possibility of traversing the energy barrier that separates the insulating and metallic states. The probability of the domain to switch back to the insulating phase is controlled by the $\left(E_{\mathrm{B}}-E_{\mathrm{c}}\right)$ difference. ${ }^{34}$

$$
P_{\mathrm{MIT}}=\mathrm{e}^{-\left(E_{\mathrm{B}}-E_{\mathrm{c}}\right) / k T}
$$

The quasi-static simulation is performed by applying a voltage across the 2-D heterogeneous resistive network and calculating the potential at each node. ${ }^{31}$ The probability that a domain undergoes an insulator to metal transition, $P_{\mathrm{IMT}}$, is then calculated using the $\Delta V$ derived from the calculated potentials at each node. On the basis of these calculated probabilities and the probability for MIT, $P_{\text {MIT }}$, the current state of the node is determined ( 0 , insulating; 1 , metallic) followed by 


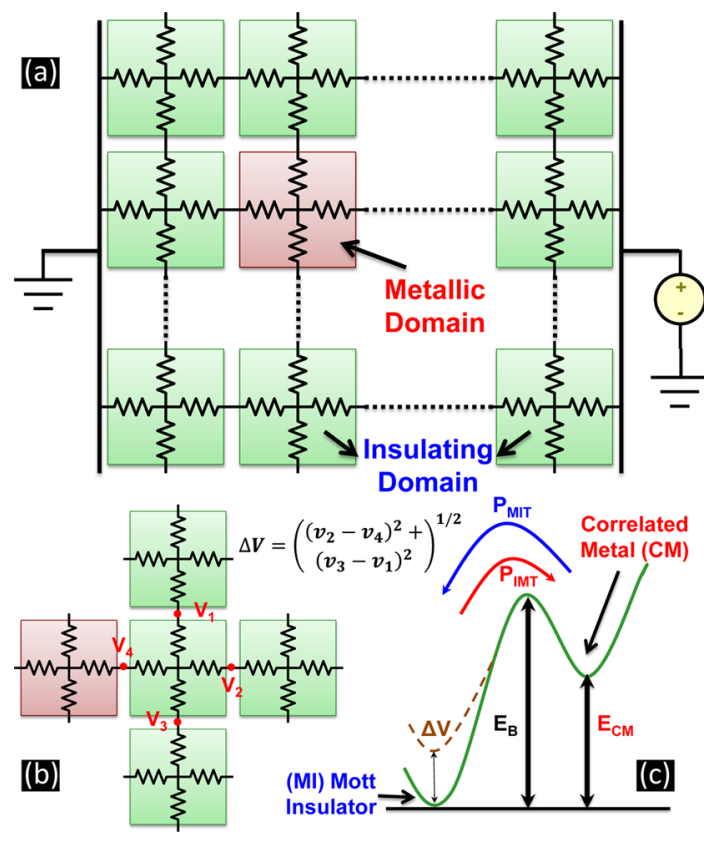

Figure 8. (a) Nanoscale domain network used to model $\mathrm{VO}_{2}$ under electrical bias. Red domains are those which have transitioned to the metallic state and green are those that remain insulating. (b) $V_{1}, V_{2}, V_{3}$, and $V_{4}$ are used to calculate $\Delta V$. (c) Energy landscape where the stable Mott insulating state and the correlated metal state are separated by an energy barrier $E_{\mathrm{B}}$.

recalculation of the potential at each node. At this point, $P_{\mathrm{IMT}}$ is re-evaluated using those potentials and the domain state determined once again. This process is repeated until the output current stabilizes. The stabilized 2D heterogeneous resistive network represents the DC state of the system in the quasi-static limit. Once this state has been determined, the voltage is stepped and the process is repeated. In this way the $I-V$ relationship can be recreated for forward and reverse voltage sweeps, as is shown in Figure 9a. The model successfully captures the abrupt nature of the IMT and closely mimics the electrical transition, including its hysteretic nature. Figure $9 \mathrm{~b}$ shows the resulting domain state at the indicated star in Figure 9a. The formation of the metallic filament across the device can be explained by the switching behavior of the domains. Considering an insulating domain surrounded by other insulating domains, the application of a large voltage across the domains will eventually cause one of the domains to switch to a lower resistance state. When this initial domain switches to become metallic, the potential drop across it is significantly reduced, which in turn is redistributed to the neighboring domains. The increase in potential across the neighboring domains due to this redistribution of field subsequently increases the probability that one of these neighboring domain also transitions to the metallic state. In this manner, a conducting filament is formed within the device channel. Due to the interplay between the domains, the formation of the filament is
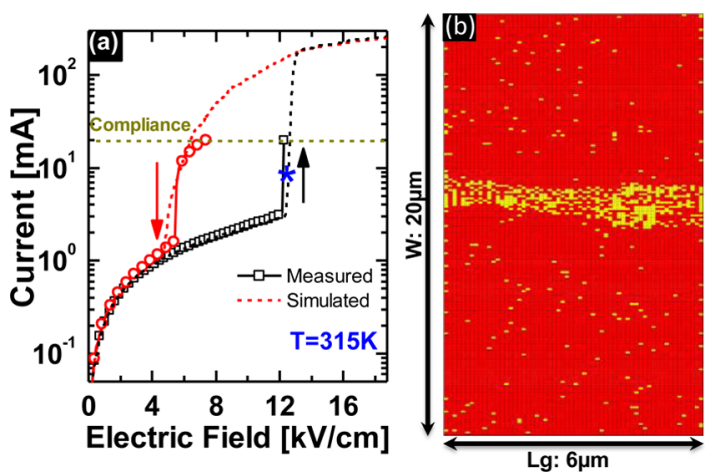

Figure 9. (a) Measured and simulated $I-V$ characteristics; measured $I-V$ was set to compliance of $20 \mathrm{~mA}$ in order to prevent device failure under high currents. (b) Simulated filamentary conduction of $6 \mu \mathrm{m}$ long and $20 \mu \mathrm{m}$ wide device (with $100 \mathrm{~nm}$ domain size) at the bias condition highlighted by asterisk $(*)$ in the $I-V$ characteristics. Metallic domains are yellow, while insulating domains appear as red.

an abrupt process. The evolution of the filament and the corresponding nodal potential can be seen in Supporting Information Figure S8. The barrier height $E_{\mathrm{B}}$ and $E_{\mathrm{C}}$ for the simulation was set to 0.55 and $0.47 \mathrm{eV}$, respectively.

It should be noted that a requirement of this calibration method is the need for effectively modeling the sample substrate system in order to get accurate $Z_{\text {tip }}$ values. So although the methodology is transferable to an arbitrary sample, the finite elemental model itself requires additional calibration to measured electrical values. This results in further measurements such as impedance spectroscopy, to accurately model the device under test. Nevertheless, the information that results from an investigation of the true nanoscale electrical properties provides valuable insight into a prototypical transition metal oxide, $\mathrm{VO}_{2}$, where the underlying transport mechanisms are still unclear.

\section{CONCLUSION AND OUTLOOK}

In summary, we employ SMM to study the dynamic phase coexistence and nanoscale domain structure of $\mathrm{VO}_{2}$ in an unstrained film during both thermal and electrically driven IMT at $16 \mathrm{GHz}$ with a spatial resolution of $50 \mathrm{~nm}$. By modeling the nanoscale domain structure as a 2-D heterogeneous resistive network, we have precisely reproduced the experimental results of the thermal and electrical phase transition. In the thermally induced IMT, the results are demonstrative of a distribution of nanoscale domains with slightly varying transition temperature. The domains in turn cluster and eventually coalesce to form total metallic coverage within the film. Further, during an electrically stimulated IMT, the presence and growth of a filamentary conducting pathway is monitored and quantitatively characterized across multiple bias points. Through implementation of the 2-D heterogeneous resistive network, it is shown that the nature of the filament formation is the result a cascading avalanche 
effect wherein the redistribution of the field across a transitioning domain upon its adjacent domains results in an abrupt switch. This work provides a quantitative framework to extract spatially resolved impedance values for a well-established IMT oxide such as $\mathrm{VO}_{2}$ or other phase transition devices as they undergo phase transition and reveal their transport and switching properties.

\section{METHODS}

Scanning Microwave Microscopy. A commercially available Agilent 5420 AFM is used with an Agilent E8364C VNA and scanning microwave nose cone assembly (N9546A-CFG001) to carry out measurements. Imaging is required to be performed in contact mode for a properly synced response between the AFM and VNA sweep at an applied in power of $5 \mathrm{dBm}$. A conductive Platinum (Pt) tip (manufactured by Rocky Mountain Nanotechnology) made from a solid conically etched Pt wire with a terminating radius of approximately $25 \mathrm{~nm}$ is affixed to a $\mathrm{Pt}$ cantilever. The cantilever is then mechanically attached to a sapphire chip completing the tip package. The tip has a height of $80 \mu \mathrm{m}$, which is significantly larger than that of coated silicon tips at $30 \mu \mathrm{m}$. A resistive heating element was controlled by a 325 Lakeshore temperature controller to maintain the temperature within $0.1 \mathrm{~K}$. For scanning microwave microscopy under bias, the sample was fabricated with long electrodes suitable for wire bonding to a chip carrier. A Keithley 4200 semiconductor characterization system was used to maintain the bias across the device in voltage mode with a specified current compliance.

Device Fabrication. The $\mathrm{VO}_{2}$ film was grown by initially performing pulsed DC mode sputtering with a solid vanadium target at 5 mTorr with $97.5 \%$ argon and $2.5 \%$ oxygen flow on $\mathrm{SiO}_{2}(100 \mathrm{~nm}) / \mathrm{Si}(675 \mu \mathrm{m})$ sample. The total sputtering time was $15 \mathrm{~min}$. This resulted in $\mathrm{VO}_{x}$ where the oxygen content $(x)$ is less than 2 . Thereafter, the vanadium film was annealed at $598 \mathrm{~K}$ for 10 min with 10 standard liters per minute (slpm) argon flow and 0.1 slpm oxygen flow. Characterization of the $\mathrm{VO}_{x}$ film using XRD showed that a single phase $\mathrm{VO}_{2}$ film was formed. The $20 \mathrm{~nm} \mathrm{Pt} / 40 \mathrm{~nm}$ Au contacts were lithographically defined and deposited by e-beam evaporation. Two terminal devices were patterned in an array with channel lengths and widths each ranging from 2 to $160 \mu \mathrm{m}$.

Conflict of Interest: The authors declare no competing financial interest.

Acknowledgment. The $\mathrm{VO}_{2}$ use in this study was fabricated at the Penn State Nanofabrication facility which is part of the National Science Foundation supported National Nanotechnology Infrastructure Network. This work was supported financially through INTEL customized SRC (Semiconductor Research Corporation) project at Penn State University and NSF MRSEC Grant DMR-08-20404 through the Center for Nanoscale Science at Penn State University. The authors also thank N. Shukla for insightful discussions, and M. Barth for sample fabrication.

Supporting Information Available: Further details on SMM operation, simulation of 2-D heterogeneous resistive network, and calibration of the COMSOL model as well as additional SMM scans during the IMT can be found in the supplementary information. This material is available free of charge via the Internet at http://pubs.acs.org.

\section{REFERENCES AND NOTES}

1. Kim, B.-J.; Lee, Y.; Choi, S.; Lim, J.-W.; Yun, S.; Kim, H.-T.; Shin, T.-J.; Yun, H.-S. Micrometer X-ray Diffraction Study of $\mathrm{VO}_{2}$ Films: Separation between Metal-Insulator Transition and Structural Phase Transition. Phys. Rev. B 2008, 77, 235401.

2. Brockman, J. S.; Gao, L.; Hughes, B.; Rettner, C. T.; Samant, M. G.; Roche, K. P.; Parkin, S. S. P. Subnanosecond Incubation Times for Electric-Field-Induced Metallization of a Correlated Electron Oxide. Nat. Nanotechnol. 2014, 9, 453-458.
3. Imada, M.; Fujimori, A.; Tokura, Y. Metal-Insulator Transitions. Rev. Mod. Phys. 1998, 70, 1039-1263.

4. Pickett, M. D.; Medeiros-Ribeiro, G.; Williams, R. S. A Scalable Neuristor Built with Mott Memristors. Nat. Mater. 2013, 12, 114-117.

5. Pickett, M. D.; Williams, R. S. Sub-100 fJ and SubNanosecond Thermally Driven Threshold Switching in Niobium Oxide Crosspoint Nanodevices. Nanotechnology 2012, 23, 215202.

6. Jeong, J.; Aetukuri, N.; Graf, T.; Schladt, T. D.; Samant, M. G.; Parkin, S. S. P. Suppression of Metal-Insulator Transition in $\mathrm{VO}_{2}$ by Electric Field-Induced Oxygen Vacancy Formation. Science 2013, 339, 1402-1405.

7. Yang, Z.; Ko, C.; Ramanathan, S. Oxide Electronics Utilizing Ultrafast Metal-Insulator Transitions. Annu. Rev. Mater. Res. 2011, 41, 337-367.

8. Cao, J.; Ertekin, E.; Srinivasan, V.; Fan, W.; Huang, S.; Zheng, H.; Yim, J. W. L.; Khanal, D. R.; Ogletree, D. F.; Grossman, J. C.; et al. Strain Engineering and One-Dimensional Organization of Metal-Insulator Domains in Single-Crystal Vanadium Dioxide Beams. Nat. Nanotechnol. 2009, 4, 732-737.

9. Muraoka, Y.; Hiroi, Z. Metal-insulator Transition of $\mathrm{VO}_{2}$ Thin Films Grown on $\mathrm{TiO}_{2}(001)$ and (110) Substrates. Appl. Phys. Lett. 2002, 80, 583.

10. Quackenbush, N. F.; Tashman, J. W.; Mundy, J. A.; Sallis, S.; Paik, H.; Misra, R.; Moyer, J. A.; Guo, J.-H.; Fischer, D. A.; Woicik, J. C.; et al. Nature of the Metal Insulator Transition in Ultrathin Epitaxial Vanadium Dioxide. Nano Lett. 2013, 13, 4857-4861.

11. Park, J. H.; Coy, J. M.; Kasirga, T. S.; Huang, C.; Fei, Z.; Hunter, S.; Cobden, D. H. Measurement of a Solid-State Triple Point at the Metal-Insulator Transition in $\mathrm{VO}_{2}$. Nature 2013, 500, 431-434.

12. Qazilbash, M. M.; Brehm, M.; Chae, B.-G.; Ho, P.-C.; Andreev, G. O.; Kim, B.-J.; Yun, S. J.; Balatsky, a V; Maple, M. B.; Keilmann, F.; et al. Mott Transition in $\mathrm{VO}_{2}$ Revealed by Infrared Spectroscopy and Nano-Imaging. Science 2007, 318, 1750-1753.

13. Cavalleri, A.; Tóth, C.; Siders, C.; Squier, J.; Ráksi, F.; Forget, P.; Kieffer, J. Femtosecond Structural Dynamics in $\mathrm{VO}_{2}$ during an Ultrafast Solid-Solid Phase Transition. Phys. Rev. Lett. 2001, 87, 237401.

14. Shukla, N.; Parihar, A.; Freeman, E.; Paik, H.; Stone, G.; Narayanan, V.; Wen, H.; Cai, Z.; Gopalan, V.; Engel-Herbert, R.; et al. Synchronized Charge Oscillations in Correlated Electron Systems. Sci. Rep. 2014, 4, 4964.

15. Dumas-Bouchiat, F.; Champeaux, C.; Catherinot, a.; Crunteanu, a.; Blondy, P. Rf-Microwave Switches Based on Reversible Semiconductor-Metal Transition of $\mathrm{VO}_{2}$ Thin Films Synthesized by Pulsed-Laser Deposition. Appl. Phys. Lett. 2007, 91, 223505.

16. Ha, S. D.; Zhou, Y.; Fisher, C. J.; Ramanathan, S.; Treadway, J. P. Electrical Switching Dynamics and Broadband Microwave Characteristics of $\mathrm{VO}_{2}$ Radio Frequency Devices. J. Appl. Phys. 2013, 113, 184501.

17. Zhang, Z.; Gao, Y.; Chen, Z.; Du, J.; Cao, C.; Kang, L.; Luo, H. Thermochromic $\mathrm{VO}_{2}$ Thin Films: Solution-Based Processing, Improved Optical Properties, and Lowered Phase Transformation Temperature. Langmuir 2010, 26, 1073810744.

18. Chang, Y.; Yang, J.; Kim, Y.; Kim, D.; Noh, T.; Kim, D.-W.; Oh, E.; Kahng, B.; Chung, J.-S. Surface versus Bulk Characterizations of Electronic Inhomogeneity in a $\mathrm{VO}_{2}$ Thin Film. Phys. Rev. B 2007, 76, 075118.

19. Freeman, E.; Stone, G.; Shukla, N.; Paik, H.; Moyer, J. a.; Cai, Z.; Wen, H.; Engel-Herbert, R.; Schlom, D. G.; Gopalan, V.; et al. 
Nanoscale Structural Evolution of Electrically Driven Insulator to Metal Transition in Vanadium Dioxide. Appl. Phys. Lett. 2013, 103, 263109.

20. Kumar, S.; Pickett, M. D.; Strachan, J. P.; Gibson, G.; Nishi, Y.; Williams, R. S. Local Temperature Redistribution and Structural Transition during Joule-Heating-Driven Conductance Switching in $\mathrm{VO}_{2}$. Adv. Mater. 2013, 25, 61286132.

21. Kanki, T.; Kawatani, K.; Takami, H.; Tanaka, H. Direct Observation of Giant Metallic Domain Evolution Driven by Electric Bias in $\mathrm{VO}_{2}$ Thin Films on $\mathrm{TiO}_{2}(001)$ Substrate. Appl. Phys. Lett. 2012, 101, 243118.

22. Tselev, A.; Lavrik, N. V.; Kolmakov, A.; Kalinin, S. V. Scanning Near-Field Microwave Microscopy of $\mathrm{VO}_{2}$ and Chemical Vapor Deposition Graphene. Adv. Funct. Mater. 2013, 23, 2635-2645.

23. Tselev, A.; Meunier, V.; Strelcov, E.; Shelton, W. a; Luk'yanchuk, I. a; Jones, K.; Proksch, R.; Kolmakov, A.; Kalinin, S. V. Mesoscopic Metal-Insulator Transition at Ferroelastic Domain Walls in $\mathrm{VO}_{2}$. ACS Nano 2010, 4, 4412-4419.

24. Wu, S.; Yu, J.-J. Attofarad Capacitance Measurement Corresponding to Single-Molecular Level Structural Variations of Self-Assembled Monolayers Using Scanning Microwave Microscopy. Appl. Phys. Lett. 2010, 97, 202902.

25. Karbassi, a; Ruf, D.; Bettermann, a D.; Paulson, C. a; van der Weide, D. W.; Tanbakuchi, H.; Stancliff, R. Quantitative Scanning near-Field Microwave Microscopy for Thin Film Dielectric Constant Measurement. Rev. Sci. Instrum. 2008, 79, 094706.

26. Huber, H. P.; Moertelmaier, M.; Wallis, T. M.; Chiang, C. J.; Hochleitner, M.; Imtiaz, a; Oh, Y. J.; Schilcher, K.; Dieudonne, M.; Smoliner, J.; et al. Calibrated Nanoscale Capacitance Measurements Using a Scanning Microwave Microscope. Rev. Sci. Instrum. 2010, 81, 113701.

27. Hoffmann, J.; Wollensack, M.; Zeier, M.; Niegemann, J.; Huber, H.-P.; Kienberger, F. A Calibration Algorithm for Nearfield Scanning Microwave Microscopes. In 2012 12th IEEE International Conference on Nanotechnology (IEEENANO); IEEE: New York, 2012; pp 1-4.

28. Gramse, G.; Kasper, M.; Fumagalli, L.; Gomila, G.; Hinterdorfer, P.; Kienberger, F. Calibrated Complex Impedance and Permittivity Measurements with Scanning Microwave Microscopy. Nanotechnology 2014, 25, 145703.

29. Zhong, X.; Zhang, X.; Gupta, A.; LeClair, P. Avalanche Breakdown in Microscale $\mathrm{VO}_{2}$ Structures. J. Appl. Phys. 2011, 110, 084516.

30. Driscoll, T.; Quinn, J.; Di Ventra, M.; Basov, D. N.; Seo, G.; Lee, Y.-W.; Kim, H.-T.; Smith, D. R. Current Oscillations in Vanadium Dioxide: Evidence for Electrically Triggered Percolation Avalanches. Phys. Rev. B 2012, 86, 094203.

31. Poklonski, N. A.; Kocherzhenko, A. A.; Benediktovitch, A. I.; Mitsianok, V. V.; Zaitsev, A. M. Simulation of Dc Conductance of Two-Dimensional Heterogeneous System: Application to Carbon Wires Made by Ion Irradiation on Polycrystalline Diamond. Phys. Status Solidi 2006, 243, 1212-1218.

32. Note, A. Agilent AN 1287-3 Applying Error Correction to Network Analyzer Measurements.

33. Comsol. Comsol Multiphysics User's Guide; Building, 2010; $\mathrm{p}$ 840.

34. Stoliar, P.; Cario, L.; Janod, E.; Corraze, B.; Guillot-Deudon, C.; Salmon-Bourmand, S.; Guiot, V.; Tranchant, J.; Rozenberg, M. Universal Electric-Field-Driven Resistive Transition in Narrow-Gap Mott Insulators. Adv. Mater. 2013, 25, 3222-3226. 